\title{
ASYMPTOTIC BIFURCATION RESULTS FOR QUASILINEAR ELLIPTIC OPERATORS
}

\author{
JAN CHABROWSKI \\ Department of Mathematics, University of Queensland, 4072, Australia \\ e-mail:jhc@maths.uq.edu.au
}

PAVEL DRÁBEK

Department of Mathematics, University of West Bohemia, Univerzitni 22, CZ-30614 Pilsen, Czech Republic e-mail:pdrabek@kma.zcu.cz

and ELLIOT TONKES

Department of Mathematics, University of Queensland, 4072, Australia

(Received 23 December, 2003; accepted 7 July, 2004)

\begin{abstract}
We develop results for bifurcation from the principal eigenvalue for certain operators based on the $p$-Laplacian and containing a superlinear nonlinearity with a critical Sobolev exponent. The main result concerns an asymptotic estimate of the rate at which the solution branch departs from the eigenspace. The method can also be applied for nonpotential operators.
\end{abstract}

2000 Mathematics Subject Classification. 47J15, 35J60, 35J65, 47J10.

1. Introduction. In this paper we investigate the nature of solutions bifurcating from the principal eigenvalue for an operator equation containing the $p$-Laplacian and its associated critical Sobolev exponent.

Let $\Omega$ be a bounded domain in $\mathbb{R}^{N}$ with smooth boundary. We seek positive solutions in the Sobolev space $W_{0}^{1, p}(\Omega)$ to the operator equation:

$$
N_{\lambda}(u) \equiv J(u)-\lambda S(u)-F_{\lambda}(u)=0,
$$

where the operators $J, S$ and $F_{\lambda}$ are defined through the duality pairing in $W_{0}^{1, p}(\Omega)$ denoted $(\cdot, \cdot)$ :

$$
\begin{aligned}
(J(u), v) & =\int_{\Omega}|\nabla u|^{p-2} \nabla u \cdot \nabla v d x \\
(S(u), v) & =\int_{\Omega}|u|^{p-2} u v d x \\
\left(F_{\lambda}(u), v\right) & =\int_{\Omega} g(x, \lambda)|u|^{p^{*}-2} u v d x,
\end{aligned}
$$

where $1<p<N$ and the critical Sobolev exponent $p^{*}=\frac{p N}{N-p}$.

The operators $J, S$ and $F_{\lambda}$ are inspired from the weak formulation of a quasilinear elliptic differential equation containing a Sobolev critical nonlinearity. Some sample 
quasilinear differential equations are later presented which can be described by the operator equation (1.1).

A vast number of publications have considered bifurcation problems in the general form of (1.1) when $J, S, F$ are various operators. The pioneering work of Krasnoselski [12] showed the existence of bifurcating solutions in Hilbert spaces when the problem is formulated with a potential. Rabinowitz [16] extended the results in Banach spaces to show that the bifurcation is a global phenomenon. Dancer [4] showed an asymptotic behaviour of the branch near the bifurcation point. More recently, Chiappinelli [3] produced an asymptotic description of the bifurcating branch, depending on the exponent in the nonlinearity. However these results all demand complete continuity of the nonlinear term $F_{\lambda}(\cdot)$. Marino [14] was successful in removing some compactness conditions from the operator.

In this paper, we formulate the problem in a Banach space, and substitute the stringent demand of complete continuity with the local $(\mathrm{S}+)$ condition. We derive local asymptotic estimates of Chiappinelli-type [3] which describe the rate at which the solution approaches the eigenspace as $\lambda$ tends to the principal eigenvalue. Our results also indicate the direction of bifurcation depending upon the interaction of the nonlinearity with the principal eigenfunction.

The estimates by Chiappinelli [3] are produced by explicitly specifying a recipe for solutions in a neighbourhood of the bifurcation point through a LusternikSchnirelmann minimax description. In our approach, the problem (1.1) is formulated directly as an operator equation, and for our working does not require the production of a potential. Our results remain valid for semilinear elliptic equations by substituting $p=2$.

As part of our solution method, the simplicity and isolatedness of the bifurcating eigenvalue is utilised, as is the positivity of the associated eigenfunction. Consequently, the results are restricted to bifurcation from the principal eigenvalue.

The linearisation of the $p$-Laplacian operator around the principal eigenvalue is used as a major tool in this paper. This technique is pioneered in [7], which treats a bifurcation from infinity to develop analogues to the Fredholm alternative. We use one of the main theorems from there.

The approach in this paper is an alternative to the variational method. For example, [1] analyses similar problems through geometric methods and arrives at existence results. However, the present approach yields further information concerning the solution.

2. Preliminaries. Assume that $\Omega \subset \mathbb{R}^{N}$ is a bounded domain with smooth boundary. Let $1<p<N$. Define $p^{*}=\frac{p N}{N-p}$, the critical Sobolev exponent. Define the usual spaces $W_{0}^{1, p}(\Omega), L^{q}(\Omega)$ with norms $\|\cdot\|_{1, p}$ and $\|\cdot\|_{q}$.

The Sobolev imbedding theorems dictate that $W_{0}^{1, p}(\Omega) \hookrightarrow L^{q}(\Omega)$, compactly if $1<q<p^{*}$ and continuously if $q=p^{*}$. The optimal coefficient in the critical imbedding is denoted $S^{*}$,

$$
S^{*}=\inf _{u \in W_{0}^{1, p}(\Omega),\|u\|_{p^{*}=1}} \int_{\Omega}|\nabla u|^{p} d x .
$$

Denote strong convergence by " $\rightarrow$ ", weak convergence by " $\rightarrow$ " and weak convergence in measure by " $\rightarrow$ *". 
By a nontrivial solution to (1.1), we mean a pair $(\lambda, u) \in \mathbb{R} \times W_{0}^{1, p}(\Omega)$ satisfying (1.1), with $u \neq 0$. The trivial solution is $(\lambda, 0)$, for any $\lambda \in \mathbb{R}$.

A point $\left(\lambda_{0}, 0\right) \in \mathbb{R} \times W_{0}^{1, p}(\Omega)$ is said to be a bifurcation point of (1.1) if there exists a sequence of nontrivial solutions $\left\{\left(\lambda_{n}, u_{n}\right)\right\}_{n=1}^{\infty}$, with $\lambda_{n} \rightarrow \lambda_{0}$ and $u_{n} \rightarrow 0$ in $W_{0}^{1, p}(\Omega)$.

Let $\mathcal{C}$ be a set in $\mathbb{R} \times W_{0}^{1, p}(\Omega)$ consisting of nontrivial solutions to (1.1) which is connected with respect to the topology induced by the norm:

$$
\|(\lambda, u)\|=\left(|\lambda|^{2}+\|u\|_{1, p}^{2}\right)^{\frac{1}{2}}
$$

Then $\mathcal{C}$ is called a continuum of nontrivial solutions of $(1.1)$. If $\left(\lambda_{0}, 0\right) \in \overline{\mathcal{C}}$, where the closure is taken with respect to the above topology, then we say that $\mathcal{C}$ bifurcates from $\left(\lambda_{0}, 0\right)$.

Let $\lambda_{1}$ be the principal eigenvalue of the $p$-Laplacian subject to Dirichlet boundary conditions on $\Omega$. It is well known that $\lambda_{1}>0$ is an isolated eigenvalue with the RayleighRitz characterisation:

$$
\lambda_{1}=\inf _{u \in W_{0}^{1, p}(\Omega), u \neq 0} \frac{\int_{\Omega}|\nabla u|^{p} d x}{\int_{\Omega}|u|^{p} d x}
$$

It is also known that $\lambda_{1}$ is simple, the corresponding normalised eigenfunction $\phi_{1}(x)$ can be taken positive in $\Omega$ and that the derivative with respect to the external normal $\partial \phi_{1} / \partial v<0$ on $\partial \Omega$ provided that the boundary is sufficiently smooth. The lower bound of the rest of the spectrum of the $p$-Laplacian is given by its second eigenvalue $\lambda_{2}>\lambda_{1}$, which also admits a variational characterisation.

For the remainder of this paper, we define convenient numbers $\underline{\lambda}$ and $\bar{\lambda}$ satisfying $0<\underline{\lambda}<\lambda_{1}<\bar{\lambda}<\lambda_{2}$, in order to derive local results around $\lambda_{1}$.

We make the following assumptions on the coefficient $g$ :

$\left(\mathrm{G}_{\infty}\right) g(x, \lambda)$ is continuous over $\bar{\Omega} \times[\underline{\lambda}, \bar{\lambda}]$, and $|g(x, \lambda)|<g_{\infty}$ for all $(x, \lambda) \in \bar{\Omega} \times[\underline{\lambda}, \bar{\lambda}]$. $\left(\mathrm{G}_{0}\right) \int_{\Omega} g\left(x, \lambda_{1}\right)\left|\phi_{1}(x)\right|^{p^{*}} d x \neq 0$.

3. Main result. Our main result is as follows:

THEOREM 3.1. Equation (1.1) admits a continuum $\mathcal{C}$ of solutions $(\lambda, u)$ in $\mathbb{R} \times$ $W_{0}^{1, p}(\Omega)$ bifurcating from $\left(\lambda_{1}, 0\right)$, and satisfying the asymptotic estimate:

$$
\frac{\lambda-\lambda_{1}}{\|u\|_{1, p}^{p^{*}-p}}=-\int_{\Omega} g\left(x, \lambda_{1}\right)\left|\phi_{1}(x)\right|^{p^{*}} d x+o(1),
$$

as $\|u\|_{1, p} \rightarrow 0$

4. Proof of the main result. We shall prove Theorem 3.1 in three stages. First we verify that there is a continuum $\mathcal{C}$ of nontrivial solutions of $(1.1)$ which bifurcates from $\left(\lambda_{1}, 0\right)$. Second we prove an $L^{\infty}$ estimate of nontrivial solutions. Third we establish the asymptotic estimate (3.5). 
4.1. Stage 1 of proof. In the first stage we verify that $\left(\lambda_{1}, 0\right)$ is a bifurcation point. The underlying technique is to show that the degree of the operator changes magnitude as $\lambda$ passes from below $\lambda_{1}$ to above.

We show that on a certain ball centred at the origin, with radius $\rho_{0}, B_{0}\left(\rho_{0}\right) \subset$ $W_{0}^{1, p}(\Omega)$, the topological degree

$$
\operatorname{deg}\left(N_{\lambda}, D, 0\right)
$$

is well defined for all open, bounded, nonempty sets $D \subset B_{0}\left(\rho_{0}\right)$, such that $N_{\lambda}(u) \neq 0$ for $u \in \partial D$. Here the degree is understood in the sense of Browder and Petryshin [2] or Skrypnik [17].

For this purpose we must prove that there exists a neighbourhood such that $N_{\lambda}$ satisfies an appropriate compactness condition, such as the $(\mathrm{S}+)$ condition from [2].

Definition 4.1. Let $X$ be a reflexive Banach space and $X^{*}$ its dual. The operator $N_{\lambda}: X \mapsto X^{*}$ is said to satisfy the local $(S+)$ condition on $D \subset X$ if any sequence $\left\{u_{n}\right\} \subset$ $D$, with $u_{n} \rightarrow u_{0}$ weakly in $X$ and $\lim _{\sup _{n \rightarrow \infty}}\left(N_{\lambda}\left(u_{n}\right), u_{n}-u_{0}\right) \leq 0$ satisfies $u_{n} \rightarrow u_{0}$ strongly in $X$.

To prove the $(\mathrm{S}+)$ condition for $N_{\lambda}$ on $B_{0}\left(\rho_{0}\right)$, we take advantage of the Concentration-Compactness Principle (CCP) of P. L. Lions [13]. The form below is taken from [9] and the Brézis-Lieb Lemma is applied to change the notation slightly.

LEMMA 4.2. Let $\left\{u_{n}\right\} \subset W_{0}^{1, p}(\Omega)$ with $\left\|u_{n}\right\|_{1, p} \leq 1, u_{n} \rightarrow u_{0}$ weakly in $W_{0}^{1, p}(\Omega)$. Then there exist measures $\mu$ and $v$ on $\bar{\Omega}$ such that:

$$
\begin{gathered}
\left|\nabla u_{n}\right|^{p} \rightarrow^{*}\left|\nabla u_{0}\right|^{p}+\mu \\
\left|u_{n}\right|^{p^{*}} \rightarrow^{*}\left|u_{0}\right|^{p^{*}}+v
\end{gathered}
$$

both weakly in the space of measures on $\bar{\Omega}$ with $v(\bar{\Omega}) \leq S^{*}(\mu(\bar{\Omega}))^{\frac{p^{*}}{p}}$. $L^{p^{*}}(\Omega)$

COROLlARY 4.3. If $\mu=0$ in Lemma 4.2, then $u_{n} \rightarrow u_{0}$ strongly both in $W_{0}^{1, p}(\Omega)$ and

Corollary 4.3 is an easy consequence of the fact that weak convergence in conjunction with convergence of the norms implies strong convergence in any uniformly convex Banach space.

Lemma 4.4. Let $\rho_{0}<\min \left\{1,\left(S^{*} g_{\infty} p\right)^{-\frac{N-p}{p^{2}}}\right\}$ and $\lambda \in[\underline{\lambda}, \bar{\lambda}]$. Then $N_{\lambda}$ satisfies the $(S+)$ condition on $B_{0}\left(\rho_{0}\right)$.

Proof. Let $\left\{u_{n}\right\}$ be a sequence in $B_{0}\left(\rho_{0}\right) \subset W_{0}^{1, p}(\Omega)$ with $u_{n} \rightarrow u_{0}$ weakly in $W_{0}^{1, p}(\Omega)$. Then $u_{n} \rightarrow u_{0}$ weakly in $L^{p^{*}}(\Omega)$ and $u_{n} \rightarrow u_{0}$ in $L^{p}(\Omega)$. We suppose that $u_{n}$ satisfies

$$
\limsup _{n \rightarrow \infty}\left(N_{\lambda}\left(u_{n}\right), u_{n}-u_{0}\right) \leq 0
$$

that is,

$$
\begin{aligned}
\limsup _{n \rightarrow \infty} & {\left[\int_{\Omega}\left|\nabla u_{n}\right|^{p-2} \nabla u_{n} \cdot \nabla\left(u_{n}-u_{0}\right) d x-\lambda \int_{\Omega}\left|u_{n}\right|^{p-2} u_{n}\left(u_{n}-u_{0}\right) d x\right.} \\
& \left.-\int_{\Omega} g(x, \lambda)\left|u_{n}\right|^{p^{*}-2} u_{n}\left(u_{n}-u_{0}\right) d x\right] \leq 0 .
\end{aligned}
$$


Let $f\left(u_{n}\right)=\left|u_{n}\right|^{p^{*}-2} u_{n}$. Then $f\left(u_{n}\right)$ is bounded in $L^{p^{*^{\prime}}}(\Omega)=L^{\frac{p N}{p N-N+p}}(\Omega)$ and hence $f\left(u_{n}\right) \rightarrow f\left(u_{0}\right)$ weakly up to a subsequence.

Since $g(\cdot, \lambda) u_{0} \in L^{p^{*}}(\Omega)$ by $\left(\mathrm{G}_{\infty}\right)$, it follows that

$$
\int_{\Omega} g(x, \lambda)\left|u_{n}\right|^{p^{*}-2} u_{n} u_{0} d x \rightarrow \int_{\Omega} g(x, \lambda)\left|u_{0}\right|^{p^{*}} d x .
$$

Using a similar argument,

$$
\int_{\Omega}\left|u_{n}\right|^{p-2} u_{n}\left(u_{n}-u_{0}\right) d x=\int_{\Omega}\left|u_{n}\right|^{p} d x-\int_{\Omega}\left|u_{0}\right|^{p} d x+o(1),
$$

but by strong convergence of $u_{n}$ in $L^{p}(\Omega)$, this vanishes as $n \rightarrow \infty$.

Thus, (4.6) becomes

$$
\begin{aligned}
\limsup _{n \rightarrow \infty} & {\left[\int_{\Omega}\left|\nabla u_{n}\right|^{p} d x-\int_{\Omega}\left|\nabla u_{n}\right|^{p-2} \nabla u_{n} \cdot \nabla u_{0} d x\right.} \\
& \left.-\int_{\Omega} g(x, \lambda)\left|u_{n}\right|^{p^{*}} d x+\int_{\Omega} g(x, \lambda)\left|u_{0}\right|^{p^{*}} d x\right] \leq 0 .
\end{aligned}
$$

By Hölder's inequality,

$$
\left.\left|\int_{\Omega}\right| \nabla u_{n}\right|^{p-2} \nabla u_{n} \cdot \nabla u_{0} d x \mid \leq\left(\int_{\Omega}\left|\nabla u_{n}\right|^{p} d x\right)^{\frac{p-1}{p}}\left(\int_{\Omega}\left|\nabla u_{0}\right|^{p} d x\right)^{\frac{1}{p}} .
$$

Now, using the CCP, we have

$$
\int_{\Omega}\left|\nabla u_{n}\right|^{p} d x \rightarrow \int_{\Omega}\left|\nabla u_{0}\right|^{p} d x+\mu(\bar{\Omega})
$$

and

$$
\int_{\Omega} g(x, \lambda)\left|u_{n}\right|^{p^{*}} d x \rightarrow \int_{\Omega} g(x, \lambda)\left|u_{0}\right|^{p^{*}} d x+\int_{\Omega} g(x, \lambda) d \nu .
$$

We may estimate $\int_{\Omega} g(x, \lambda) d v \leq g_{\infty} \int_{\Omega} d v=g_{\infty} v(\bar{\Omega})$. Thus, (4.6) now becomes

$$
\begin{aligned}
0 & \geq \int_{\Omega}\left|\nabla u_{0}\right|^{p} d x+\mu(\bar{\Omega})-\limsup _{n \rightarrow \infty}\left(\int_{\Omega}\left|\nabla u_{n}\right|^{p} d x\right)^{\frac{p-1}{p}}\left(\int_{\Omega}\left|\nabla u_{0}\right|^{p} d x\right)^{\frac{1}{p}}-g_{\infty} v(\bar{\Omega}) \\
& \geq \int_{\Omega}\left|\nabla u_{0}\right|^{p} d x+\mu(\bar{\Omega})-\left(\int_{\Omega}\left|\nabla u_{0}\right|^{p} d x+\mu(\bar{\Omega})\right)^{\frac{p-1}{p}}\left(\int_{\Omega}\left|\nabla u_{0}\right|^{p} d x\right)^{\frac{1}{p}}-S^{*} g_{\infty} \mu(\bar{\Omega})^{\frac{p^{*}}{p}} .
\end{aligned}
$$

For brevity, let $\int_{\Omega}\left|\nabla u_{0}\right|^{p} d x=a^{p}$ and denote $\mu(\bar{\Omega})$ and $v(\bar{\Omega})$ by $\mu$ and $v$, respectively. Then we can write (4.7) as

$$
h(\mu)=a^{p}+\mu-\left(a^{p}+\mu\right)^{\frac{p-1}{p}} a-S^{*} g_{\infty} \mu^{\frac{p^{*}}{p}} \leq 0 .
$$

We have $a^{p}+\mu \leq \lim \sup _{n \rightarrow \infty}\left\|u_{n}\right\|_{1, p}^{p} \leq \rho_{0}^{p}<1$. Hence $\mu<1$. Thus, expression (4.8) is equivalent to finding $\mu \in[0,1)$ satisfying $h(\mu) \leq 0$.

It can easily be checked that $\left(a^{p}+\mu\right)^{\frac{p-1}{p}} a \leq a^{p}+\frac{p-1}{p} \mu$ for $0 \leq \mu, a^{p}<1$ and $p>1$. 
Hence

$$
\tilde{h}(\mu)=a^{p}+\mu-a^{p}-\frac{p-1}{p} \mu-S^{*} g_{\infty} \mu^{\frac{p^{*}}{p}}=\frac{1}{p} \mu-S^{*} g_{\infty} \mu^{\frac{p^{*}}{p}} \leq h(\mu) .
$$

But if $\tilde{h}(\mu) \leq 0$, then it follows directly that $\mu=0$ or

$$
\mu \geq\left(S^{*} g_{\infty} p\right)^{-\frac{N-p}{p}}
$$

and $\mu$ satisfying $h(\mu) \leq 0$ must be bounded by the same constant. Since the assumptions of the theorem demand that $\left\|u_{n}\right\|_{1, p}<\rho_{0}$, it must follow that $\mu<\rho_{0}^{p}<\left(S^{*} g_{\infty} p\right)^{-\frac{N-p}{p}}$ but this contradicts (4.9). Hence, $\mu(\bar{\Omega})=0$ and due to Lemma 4.2 it follows that also $v(\bar{\Omega})=0$, no concentration takes place and $u_{n} \rightarrow u_{0}$ in $W_{0}^{1, p}(\Omega)$.

In fact, this argument is valid only to verify strong convergence of a subsequence. The fact that the full sequence must converge strongly is easily proved by contradiction. Suppose that for some subsequence $\left\{u_{n_{k}}\right\}_{k=1}^{\infty} \subset\left\{u_{n}\right\}_{n=1}^{\infty}$ it holds that $u_{n_{k}} \rightarrow u_{0}$ weakly in $W_{0}^{1, p}(\Omega)$ and

$$
\limsup _{k \rightarrow \infty}\left(N_{\lambda}\left(u_{n_{k}}\right), u_{n_{k}}-u_{0}\right) \leq 0
$$

but $u_{n_{k}} \not \rightarrow u_{0}$ in $W_{0}^{1, p}(\Omega)$. Then we have $\left\|u_{n_{k}}-u_{0}\right\|_{1, p} \geq \delta$ with some $\delta>0$ for a subsequence again labelled $u_{n_{k}}$. Proceeding as above, we can prove that there is a strongly convergent subsequence of $u_{n_{k}}$, yielding a contradiction. This completes the proof.

In particular, it follows from Lemma 4.4 that the degree, $\operatorname{deg}\left(N_{\lambda}, B_{0}(\rho), 0\right)$ is well defined for any $0<\rho<\rho_{0}$ whenever $0 \notin N_{\lambda}\left(\partial B_{0}(\rho)\right)$.

Let

$$
\tilde{N}_{\lambda}(u)=J(u)-\lambda S(u)
$$

Clearly, for $\lambda \neq \lambda_{1}, \lambda \in[\underline{\lambda}, \bar{\lambda}]$, the degree $\operatorname{deg}\left(\tilde{N}_{\lambda}, B_{0}(\rho), 0\right)$ is well defined. Moreover, it follows from Drábek, Kufner and Nicolosi [6] (pp. 149-152) that for any $0<\delta<\bar{\lambda}-\lambda_{1}$ we have

$$
\operatorname{deg}\left(\tilde{N}_{\lambda_{1}-\delta}, B_{0}(\rho), 0\right)=1 \text {, and } \operatorname{deg}\left(\tilde{N}_{\lambda_{1}+\delta}, B_{0}(\rho), 0\right)=-1 .
$$

The assumption $\left(\mathrm{G}_{0}\right)$ implies that

$$
\lim _{\|u\|_{1, p} \rightarrow 0} \frac{\left\|F_{\lambda}(u)\right\|_{-1, p^{\prime}}}{\|u\|_{1, p}^{p-1}}=0
$$

uniformly for all $\lambda \in[\underline{\lambda}, \bar{\lambda}]$. Then (4.10), (4.11) and the homotopy invariance property of the degree yield that for any $0<\delta<\bar{\lambda}-\lambda_{1}$ there exists $\rho>0$ such that

$$
\operatorname{deg}\left(N_{\lambda_{1}-\delta}, B_{0}(\rho), 0\right)=1, \text { and } \operatorname{deg}\left(N_{\lambda_{1}+\delta}, B_{0}(\rho), 0\right)=-1 .
$$

In other words, the index of the isolated zero of $N_{\lambda}$ changes by magnitude 2 when $\lambda$ crosses $\lambda_{1}$. Following literally the proof of Theorem 1.3 and Corollary 1.12 in Rabinowitz [16] we then have: 
Proposition 4.5. Equation (1.1) admits a continuum $\mathcal{C}$ of nontrivial solutions $(\lambda, u) \subset \mathbb{R} \times W_{0}^{1, p}(\Omega)$ bifurcating from $\left(\lambda_{1}, 0\right)$. The continuum meets the boundary of $[\underline{\lambda}, \bar{\lambda}] \times B_{0}\left(\rho_{0}\right)$.

4.2. Stage 2 of proof. The next stage of the proof is to obtain an $L^{\infty}$ bound for nontrivial solutions from Proposition 4.5. In order to utilise the main bifurcation theorem from [7] we must verify that nontrivial solutions $(\lambda, u) \in \mathcal{C}$ are uniformly bounded in $L^{\infty}(\Omega)$ provided that $\|u\|_{1, p}$ is sufficiently small.

To achieve this, we implement Moser iterations [15], and follow a style of proof from Proposition 1.2 in Guedda and Veron [10]. Adaptations are made to suit the particular structures assumed in (1.1).

LEMmA 4.6. There exists $0<\rho_{1}<\rho_{0}$ such that for any $t \in[1, \infty)$ and for any $(\lambda, u) \in \mathcal{C}$ with $\|u\|_{1, p} \leq \rho_{1}$, and $\underline{\lambda} \leq \lambda \leq \bar{\lambda}$, there exists a constant $c(t)>0$ depending on $t,|\Omega|, N$, p and $g_{\infty}$ such that $\|u\|_{t} \leq c(t)$.

Proof. Consider all solutions to (1.1) which lie in $[\lambda, \bar{\lambda}] \times B_{0}\left(\rho_{0}\right)$. Suppose that $(\tilde{\lambda}, \tilde{u})$ is such a solution. It must follow then that $\tilde{u}$ is a solution of the following problem (in the unknown function $u(x) \in W_{0}^{1, p}(\Omega)$ ):

$$
-\nabla \cdot\left(|\nabla u|^{p-2} \nabla u\right)+K_{\tilde{u}}(x)|u|^{p-2} u=f_{\tilde{u}}(x)
$$

where

$$
\begin{aligned}
K_{\tilde{u}}(x) & =-\operatorname{sgn}(\tilde{u}) \frac{\tilde{\lambda}|\tilde{u}|^{p-2} \tilde{u}+g(x, \lambda)|\tilde{u}|^{p^{*}-2} \tilde{u}}{1+|\tilde{u}|^{p-1}} \\
f_{\tilde{u}}(x) & =\operatorname{sgn}(\tilde{u}) K_{\tilde{u}}(x) .
\end{aligned}
$$

Under the assumption $\left(\mathrm{G}_{\infty}\right)$ it holds that there exist constants $C^{\prime}$ and $D^{\prime}$ depending on $\bar{\lambda}$ and $g_{\infty}$ such that

$$
\left|K_{\tilde{u}}(x)\right| \leq C^{\prime}|\tilde{u}|^{p^{*}-p}+D^{\prime}
$$

Following the ideas in [10] (pp. 882-883), define $h, \phi \in C^{1}$ for $k>0$ and $t \geq p$ by:

$$
h(r)= \begin{cases}\operatorname{sgn}(r)|r|^{t / p}, & \text { if }|r| \leq k \\ \operatorname{sgn}(r) \frac{t}{p} k^{\frac{t}{p}-1}|r|+\left(1-\frac{t}{p}\right) k^{\frac{t}{p}}, & \text { if }|r|>k\end{cases}
$$

and $\phi(r)=\int_{0}^{r}\left|h^{\prime}(s)\right|^{p} d s$. Then $\phi(u) \in W_{0}^{1, p}(\Omega)$ and testing (4.12) with $\phi(u)$, we obtain:

$$
\int_{\Omega}|\nabla h(u)|^{p} d x+\int_{\Omega} K_{\tilde{u}} \phi(u)|u|^{p-2} u d x=\int_{\Omega} f_{\tilde{u}} \phi(u) d x,
$$

giving

$$
\left.\left|\int_{\Omega}\right| \nabla h(u)\right|^{p} d x|\leq| \int_{\Omega} K_{\tilde{u}} \phi(u)|u|^{p-2} u d x|+| \int_{\Omega} f_{\tilde{u}} \phi(u) d x \mid .
$$


The first term in (4.14) can be estimated with the Sobolev inequality to give

$$
\int_{\Omega}|\nabla h(u)|^{p} d x \geq S^{*}\left(\int_{\Omega}|h(u)|^{p^{*}} d x\right)^{\frac{p}{p^{*}}} .
$$

The second term in (4.14) can be approximated using (4.13) along with Hölder's inequality to give

$$
\begin{aligned}
& \int_{\Omega} K_{\tilde{u}} \phi(u)|u|^{p-2} u d x \\
& \quad \leq C^{\prime}\left(\int_{\Omega}|\tilde{u}|^{p^{*}} d x\right)^{\frac{p}{N}}\left(\left.\left.\int_{\Omega}|| u\right|^{p-1} \phi(u)\right|^{\frac{N}{N-p}} d x\right)^{\frac{N-p}{N}}+\left.D^{\prime} \int_{\Omega}|\phi(u)| u\right|^{p-1} \mid d x .
\end{aligned}
$$

From the definition of $h$ and $\phi$, there exits $C$ independent of $k$ such that

$$
\left.\left.|| r\right|^{p-1} \phi(r)|\leq C| h(r)\right|^{p}, \quad|\phi(r)| \leq C|h(r)|^{p(t+1-p) / t} .
$$

Thus, using the first inequality in (4.15), we can write

$$
\int_{\Omega} K_{\tilde{u}} \phi(u)|u|^{p-2} u d x \leq C C^{\prime}\left(\int_{\Omega}|\tilde{u}|^{p^{*}} d x\right)^{\frac{p}{N}}\left(\int_{\Omega}|h(u)|^{p^{*}} d x\right)^{\frac{p}{p^{*}}}+C D^{\prime} \int_{\Omega}|h(u)|^{p} d x .
$$

Since $\|\tilde{u}\|_{p^{*}}^{\frac{p}{p^{*}}} \leq S^{*}\|\tilde{u}\|_{1, p}$, we choose $\rho_{1}$ sufficiently small that for all $\|v\|_{1, p} \leq \rho_{1}$,

$$
C C^{\prime}\left(\int_{\Omega}|v|^{p^{*}} d x\right)^{\frac{p}{N}} \leq \frac{S^{*}}{2} .
$$

By Hölder's inequality and the second inequality in (4.15), the third term in (4.14) can be estimated by

$$
\begin{aligned}
\int_{\Omega} f_{\tilde{u}} \phi(u) d x & \leq C\left\|f_{\tilde{u}}\right\|_{\frac{N}{p}}\left(\int_{\Omega}|h(u)|^{p^{*}(t+1-p) / t} d x\right)^{\frac{p}{p^{*}}} \\
& \leq C\left\|f_{\tilde{u}}\right\|_{\frac{N}{p}}|\Omega|^{p(p-1) /\left(p^{*} t\right)}\left(\int_{\Omega}|h(u)|^{p^{*}} d x\right)^{(t+1-p) p /\left(p^{*}\right)} .
\end{aligned}
$$

Combining these estimates, (4.14) becomes

$$
\begin{aligned}
& \frac{S^{*}}{2}\left(\int_{\Omega}|h(u)|^{p^{*}} d x\right)^{\frac{p}{p^{*}}} \\
& \quad \leq D^{\prime} C \int_{\Omega}|h(u)|^{p} d x+C\left\|f_{\tilde{u}}\right\|_{\frac{N}{p}}|\Omega|^{p(p-1) /\left(p^{*} t\right)}\left(\int_{\Omega}|h(u)|^{p^{*}} d x\right)^{(t+1-p) p /\left(p^{*}\right)} .
\end{aligned}
$$

Assuming that $u \in L^{t}(\Omega)$, and taking passage to the limit as $k \rightarrow \infty$, we have

$$
\frac{S^{*}}{2}\|u\|_{\frac{t N}{N-p}}^{t} \leq D^{\prime} C\|u\|_{t}^{t}+C\left\|f_{\tilde{u}}\right\|_{\frac{N}{p}}|\Omega|^{p(p-1) /\left(p^{*} t\right)}\|u\|_{\frac{t N}{N-p}}^{t+1-p}
$$


and with Young's inequality, for any $\delta>0$,

$\frac{S^{*}}{2}\|u\|_{\frac{t N}{N-p}}^{t} \leq D^{\prime} C\|u\|_{t}^{t}+\frac{p-1}{t}\left(C|\Omega|^{p(p-1) /\left(p^{*} t\right)}\left\|f_{\tilde{u}}\right\|_{\frac{N}{p}} \delta\right)^{\frac{t}{p-1}}+\frac{1}{\delta^{t /(t+1-p)}} \frac{t+1-p}{t}\|u\|_{\frac{t N}{N-p}}^{t}$.

With appropriate choice of $\delta>0$, we have that

$$
\|u\|_{\frac{t N}{N-p}} \leq \alpha\|u\|_{t}+\beta^{\prime}\left\|f_{\tilde{u}}\right\|_{\frac{N}{p}}^{\frac{1}{p-1}}
$$

where $\alpha$ and $\beta^{\prime}$ depend on $t, N, p,|\Omega|$. Further, $\left\|f_{\tilde{u}}\right\|_{\frac{N}{p}} \leq C^{\prime}\|\tilde{u}\|_{p^{*}}^{\frac{p}{N}}+D^{\prime}|\Omega|^{\frac{p}{N}}$, and since we consider only small solutions, a uniform bound is imposed on $\|\tilde{u}\|_{p^{*}}$. Consequently, we have

$$
\|u\|_{\frac{t N}{N-p}} \leq \alpha\|u\|_{t}+\beta
$$

where $\alpha, \beta$ depend on $t, N, p,|\Omega|$ and $g_{\infty}$.

The procedure outlined above now iterates to establish that $\|u\|_{t} \leq c(t)$ for any $t>1$. Indeed, we may commence with $t=p^{*}$, as $u$ is uniformly bounded in $W_{0}^{1,2}(\Omega)$, and hence also in $L^{p^{*}}(\Omega)$. Repeating the argument gives a bound for $\|u\|_{t_{l}}$, for $t_{l}=\left(\frac{N}{N-p}\right)^{l} p$, and any positive integer $l$.

We remark that the main departure of Lemma 4.6 from the original proposition $1.2 \mathrm{in} \mathrm{[10]}$ is to eliminate the dependence of $c(t)$ on $K$ by using knowledge of the specific form of $K_{\tilde{u}}$, and the restriction to small $\tilde{u}$ in $W_{0}^{1, p}(\Omega)$.

Lemma 4.7. There exists $c=c\left(\rho_{1}\right)>0$ such that for any $(\lambda, u) \in \mathcal{C}$ with $\|u\|_{1, p} \leq \rho_{1}$ and $\underline{\lambda} \leq \lambda \leq \bar{\lambda}$, we have $\|u\|_{\infty} \leq c$.

Proof. If $(\tilde{\lambda}, \tilde{u})$ is a solution of (1.1), then $\tilde{u}$ must solve (in the unknown function $u(x))$ :

$$
-\nabla \cdot\left(|\nabla u|^{p-2} \nabla u\right)=G_{\tilde{u}}(x)
$$

where

$$
G_{\tilde{u}}(x)=\tilde{\lambda}|\tilde{u}|^{p-2} \tilde{u}+g(x, \tilde{\lambda})|\tilde{u}|^{p^{*}-2} \tilde{u}
$$

From the previous lemma for any solution of (1.1) with $\|\tilde{u}\|_{1, p} \leq \rho_{1}$, we have $\left\|G_{\tilde{u}}(x)\right\|_{s} \leq$ $C(s)$ for an arbitrary $s>\frac{N}{p}$. The proof now follows directly by implementing Moser iterations [15] similarly to Proposition 1.3 of [10]. Since the dependence of $C(s)$ on $\tilde{u}$ is only through $\|\tilde{u}\|_{1, p}$, the bound $\|u\|_{\infty} \leq c$ holds for all solutions with $\|u\|_{1, p} \leq \rho_{1}$.

REMARK 4.8. Corollary 1.1 from [10] actually goes further, and verifies some regularity of the solution. For some $\alpha \in(0,1)$ we have that $u \in C^{1, \alpha}(\bar{\Omega})$ for any $(\lambda, u) \in$ $\mathcal{C},\|u\|_{1, p} \leq \rho_{1}$.

4.3. Stage 3 of proof. Next we apply an asymptotic estimate from Theorem 4.1 in Drábek, Girg, Takác and Ulm [7]. In fact, only part of the estimate is required, and it is presented explicitly here for the convenience of the reader. We will occasionally use the subspace $\left(W_{0}^{1, p}\right)^{T}=\left\{v \in W_{0}^{1, p}(\Omega): \int_{\Omega} v \phi_{1} d x=0\right\}$. 
LemmA 4.9. Let $\left\{\alpha_{n}\right\}_{n=1}^{\infty} \subset \mathbb{R},\left\{\tilde{f}_{n}\right\}_{n=1}^{\infty} \subset L^{\infty}(\Omega),\left\{\tilde{u}_{n}\right\}_{n=1}^{\infty} \subset W_{0}^{1, p}(\Omega)$ be sequences such that

1. $\lambda_{1}+\alpha_{n}<\bar{\lambda}$ for all $n \in \mathbb{N}$,

2. $\tilde{f}_{n} \rightarrow^{*} \tilde{f}$ weakly-star in $L^{\infty}(\Omega)$,

3. $\left\|\tilde{u}_{n}\right\|_{1, p} \rightarrow \infty$ as $n \rightarrow \infty$,

4. assume that for all $n \in \mathbb{N}$ and $\psi \in W_{0}^{1, p}(\Omega)$,

$$
\int_{\Omega}\left|\nabla \tilde{u}_{n}\right|^{p-2} \nabla \tilde{u}_{n} \cdot \nabla \psi d x=\left(\lambda_{1}+\alpha_{n}\right) \int_{\Omega}\left|\tilde{u}_{n}\right|^{p-2} \tilde{u}_{n} \psi d x+\int_{\Omega} \tilde{f}_{n} \psi d x .
$$

Then $\alpha_{n} \rightarrow 0$ and, writing $\tilde{u}_{n}=t_{n}^{-1}\left(\phi_{1}+v_{n}^{T}\right)$, with $t_{n} \in \mathbb{R}, t_{n} \neq 0$ and $v_{n}^{T} \in\left(W_{0}^{1, p}\right)^{T}$, we have $t_{n} \rightarrow 0, v_{n}^{T} \rightarrow 0$ in $C^{1, \alpha}(\bar{\Omega}), \alpha \in(0,1)$ and

$$
\alpha_{n}=-\left|t_{n}\right|^{p-2} t_{n} \int_{\Omega} \tilde{f}_{n} \phi_{1} d x+o\left(\left|t_{n}\right|^{p-1}\right)
$$

as $n \rightarrow \infty$.

We confirm that the expression of the present operator $N_{\lambda}$ can be adapted to satisfy the criteria imposed by Lemma 4.9. Let $\left(\lambda_{n}, u_{n}\right) \in \mathcal{C},\left\|u_{n}\right\|_{1, p} \rightarrow 0$, and decompose $\lambda_{n}$ and $u_{n}$ as follows: $\lambda_{n}=\lambda_{1}+\alpha_{n}, u_{n}=\tau_{n}\left(\phi_{1}+v_{n}^{T}\right), \tau_{n} \in \mathbb{R}, v_{n}^{T} \in\left(W_{0}^{1, p}\right)^{T}$.

Since any solution $u$ has a counterpart solution $-u$, we may assume without loss of generality that $\tau_{n}>0$. Then $\tau_{n} \rightarrow 0$ and

$$
\begin{aligned}
& \tau_{n}^{p-1} \int_{\Omega}\left|\nabla \phi_{1}+\nabla v_{n}^{T}\right|^{p-2}\left(\nabla \phi_{1}+\nabla v_{n}^{T}\right) \cdot \nabla \psi d x \\
& =\left(\lambda_{1}+\alpha_{n}\right) \tau_{n}^{p-1} \int_{\Omega}\left|\phi_{1}+v_{n}^{T}\right|^{p-2}\left(\phi_{1}+v_{n}^{T}\right) \psi d x \\
& \quad+\tau_{n}^{p^{*}-1} \int_{\Omega} g\left(x, \lambda_{1}+\alpha_{n}\right)\left|\phi_{1}+v_{n}^{T}\right|^{p^{*}-2}\left(\phi_{1}+v_{n}^{T}\right) \psi d x .
\end{aligned}
$$

It is straightforward to verify that (4.19) is identical to (4.17), where $\tilde{u}_{n}=t_{n}^{-1}\left(\phi_{1}+\right.$ $\left.v_{n}^{T}\right), t_{n}=\tau_{n}^{\frac{p^{*}-p}{p-1}}, \tilde{f}_{n}=g\left(x, \lambda_{1}+\alpha_{n}\right)\left|\phi_{1}+v_{n}^{T}\right|^{p^{*}-2}\left(\phi_{1}+v_{n}^{T}\right)$.

By $\left(\mathrm{G}_{\infty}\right)$, Lemma 4.7 and rescaling argument, we have that $\left\{\tilde{f}_{n}\right\}_{n=1}^{\infty}$ is a bounded sequence in $L^{\infty}(\Omega)$. Passing to a subsequence, we can assume that $\tilde{f}_{n} \rightarrow^{*} \tilde{f}$ weaklystar in $L^{\infty}(\Omega)$. We also have $\left\|\tilde{u}_{n}\right\|_{1, p} \rightarrow \infty$. It follows from Lemma 4.9 that $v_{n}^{T} \rightarrow 0$ in $C^{1, \alpha}(\bar{\Omega})$ and so (4.18) holds. In our notation,

$$
\tilde{f}(x)=g\left(x, \lambda_{1}\right)\left|\phi_{1}(x)\right|^{p^{*}-2} \phi_{1}(x)
$$

by $\left(\mathrm{G}_{0}\right)$ and the uniform convergence $v_{n}^{T} \rightarrow 0$ in $\bar{\Omega}$. Then (4.18) is equivalent to

$$
\frac{\alpha_{n}}{\left|t_{n}\right|^{p-2} t_{n}}=-\int_{\Omega} g\left(x, \lambda_{1}\right)\left|\phi_{1}(x)\right|^{p^{*}} d x+o(1) .
$$

That is, in terms of $\tau_{n}$ and $\lambda_{n}$,

$$
\frac{\lambda_{n}-\lambda_{1}}{\tau_{n}^{p^{*}-p}}=-\int_{\Omega} g\left(x, \lambda_{1}\right)\left|\phi_{1}(x)\right|^{p^{*}} d x+o(1)
$$

as $n \rightarrow \infty$. 


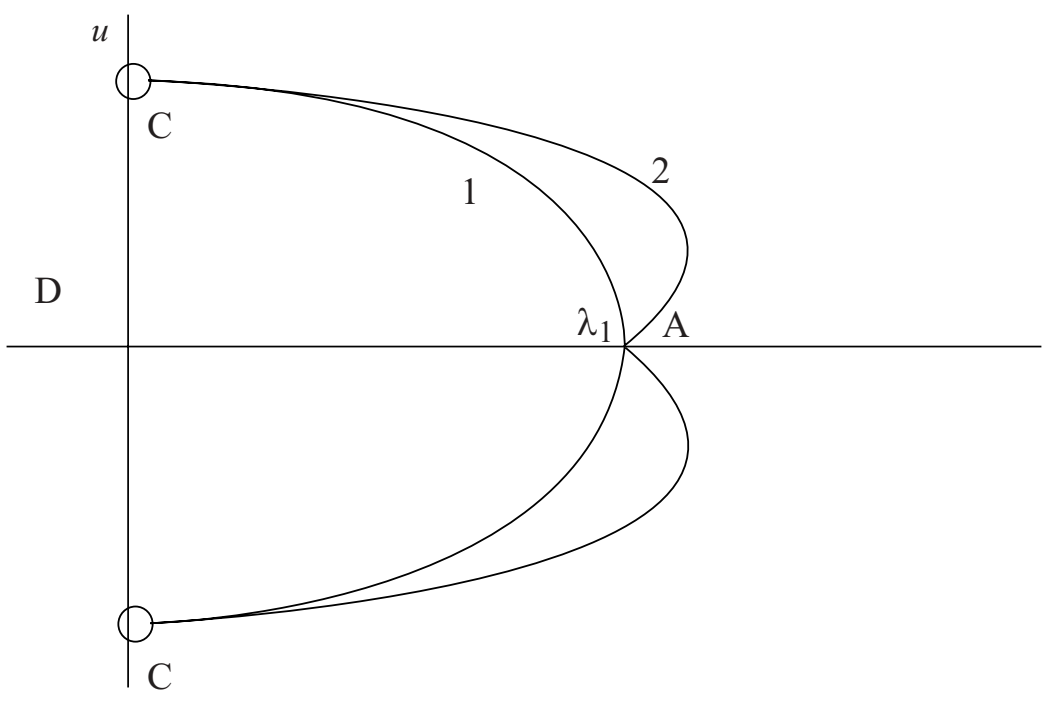

Figure 1 Stylised bifurcation diagram for (1.1)

A similar argument to that used in the proof of Lemma 4.4 implies that (4.20) holds true for any $\left(\lambda_{n}, u_{n}\right) \in \mathcal{C}$, and not only for a subsequence.

Since $\left\|u_{n}\right\|_{1, p}=\tau_{n}+o\left(\tau_{n}\right)$ as $n \rightarrow \infty$, due to the uniform convergence of $v_{n}^{T} \rightarrow 0$ in $\bar{\Omega}$, expression (3.5) follows from (4.20). We also have that $u_{n}(x)>0$ in $\Omega$ if $n$ is sufficiently large. This completes the proof of Theorem 3.1 .

5. Concluding remarks and examples. The problem (1.1) is inspired by differential equations containing the $p$-Laplacian. Weak positive solutions to the differential equation,

$$
-\Delta_{p} u-\lambda u^{p}-g(x, \lambda)|u|^{p^{*}-2} u=0
$$

on $\Omega \subset \mathbb{R}^{N}$ with homogeneous Dirichlet boundary conditions correspond with solutions to (1.1). Solutions bifurcate from $\left(\lambda_{1}, 0\right)$ and branch to the left or right depending on the sign of $\int_{\Omega} g\left(x, \lambda_{1}\right)\left|\phi_{1}(x)\right|^{p^{*}} d x$. The evidence in [8] found that, depending on the sign of $\int_{\Omega} g\left(x, \lambda_{1}\right)\left|\phi_{1}(x)\right|^{p^{*}} d x$, there may be two solutions in a right neighbourhood of $\lambda_{1}$, or one solution in a left neighbourhood of $\lambda_{1}$ and none in a right neighbourhood. For $g \equiv 1$, Azorero and Alonso [1] use the variational approach to find that at each $0<\lambda<\lambda_{1}$, there is a nontrivial solution to the problem. They find estimates of the associated energy functional depending on $\lambda$ which are consistent with our derived magnitudes of $\|u\|_{1, p}$. With $p=2$, asymptotic estimates of the bifurcation branch are consistent with the Chiappinelli estimates [3].

Accumulated evidence of this type of problem now points to behaviour of the solution according to the stylised bifurcation diagram of Figure 1.

Curve 1 (resp. 2) represents the bifurcation branch if $\int_{\Omega} g\left(x, \lambda_{1}\right)\left|\phi_{1}(x)\right|^{p^{*}} d x>0$ (resp. $<0)$. Region $\mathbf{A}$ is described by the asymptotic estimate from Theorem 3.1. The reflected solution demonstrates that there are positive and negative solutions by symmetry. Region $\mathbf{C}$ represents solutions which concentrate as $\lambda \rightarrow 0$ at peaks of $g$ in accordance 
with [11]. Region $\mathbf{D}$ describes the nonexistence of positive solutions for $\lambda<0$ by Pohozaev's nonexistence result.

In accordance with Corollary 1.12 of [16], global bifurcation results may fail when compactness is lost. In our case, a critical exponent yields only a local ( $\mathrm{S}+$ ) compactness condition (as weakly convergent sequences with certain magnitudes may concentrate to atoms of measure). This behaviour is consistent with a continuum of solutions which evaporates at Region $\mathbf{C}$ when there is a failure of compactness. Although there are strong resemblances between the local $(\mathrm{S}+)$ and the familiar local Palais-Smale conditions, and they are verified using similar techniques, a relationship between the two does not seem to be directly expressible.

The mechanics of our result can be extended to nonvariational problems also. The functional-differential equation:

$$
-\Delta_{p} u-\lambda u^{p}-g\left(x, \lambda,\|u\|_{1, p}\right) u^{p^{*}-1}=0
$$

with Dirichlet boundary conditions, is expressed in weak form by (1.1), where

$$
\left(F_{\lambda}(u), v\right)=\int_{\Omega} g\left(x, \lambda,\|u\|_{1, p}\right)|u|^{p^{*}-2} u v d x .
$$

Assume that $g(x, \lambda, s)$ is continuous on $\bar{\Omega} \times[\underline{\lambda}, \bar{\lambda}] \times[0, \infty)$ and bounded in magnitude by $g_{\infty}$.

The main result (1.1) remains valid with the obvious changes in notation. Condition $\left(\mathrm{G}_{0}\right)$ becomes $\int_{\Omega} g(x, \lambda, s)\left|\phi_{1}(x)\right|^{p^{*}} d x \neq 0$ for $\lambda \in[\underline{\lambda}, \bar{\lambda}]$ and $0 \leq s<s_{0}$, for some $s_{0}$.

The proof of Lemma 4.4 proceeds as before, but noting that $\left\{u_{n}\right\}$ is bounded in $W_{0}^{1, p}(\Omega)$, it follows that a subsequence $\left\|u_{n}\right\|_{1, p}$ converges to, say, $\xi_{0}$. Now, $g\left(x, \lambda,\left\|u_{n}\right\|_{1, p}\right)$ converges uniformly over $\bar{\Omega}$, yielding

$$
\begin{aligned}
\int_{\Omega} g\left(x, \lambda,\left\|u_{n}\right\|_{1, p}\right)\left|u_{n}\right|^{p^{*}} d x & =\int_{\Omega} g\left(x, \lambda, \xi_{0}\right)\left|u_{n}\right|^{p^{*}} d x+o(1), \text { and } \\
\int_{\Omega} g\left(x, \lambda,\left\|u_{n}\right\|_{1, p}\right)\left|u_{n}\right|^{p^{*}-2} u_{n} u_{0} d x & =\int_{\Omega} g\left(x, \lambda, \xi_{0}\right)\left|u_{n}\right|^{p^{*}-2} u_{n} u_{0} d x+o(1) .
\end{aligned}
$$

The remainder of the proof follows the previous argument, confirming that $u_{n} \rightarrow u_{0}$ in $W_{0}^{1, p}(\Omega)$, and establishing that $\xi_{0}=\left\|u_{0}\right\|_{1, p}$.

ACKNOWLEDGement. The second author acknowledges the support of the E. Raybould Fellowship and the Grant Agency of the Czech Republic, grant number 201/03/0671.

\section{REFERENCES}

1. J. P. Garcia Azorero and I. Peral Alonso, Existence and nonuniqueness for the pLaplacian: nonlinear eigenvalues, Commun. Partial Differential Equations 12 (1987), 1389-1430.

2. F. E. Browder and W. V. Petryshin, Approximation methods and the generalised topological degree for nonlinear mappings in Banach spaces, J. Func. Anal. 3 (1969), 217-245.

3. R. Chiappinelli, An estimate on the eigenvalues in bifurcation for gradient mappings, Glasgow Math. J. 39 (1997), 211-216. 
4. E. N. Dancer, Bifurcation theory in real Banach space, Proc. London Math. Soc. (3) 23 (1971), 699-734.

5. P. Drábek, Solvability and bifurcations of nonlinear equations (Longman, 1992).

6. P. Drábek, A. Kufner and F. Nicolosi, Quasilinear elliptic equations with degenerations and singularities (Walter de Gruyter, Berlin, 1997).

7. P. Drábek, P. Girg, P. Takác and M. Ulm, The Fredholm alternative for the $p$-Laplacian: bifurcation from infinity, existence and multiplicity, Indiana Univ. Math. J. 53 (2004), 433-482.

8. P. Drábek and Y. X. Huang, Multiplicity of positive solutions for some quasilinear elliptic equation in $\mathbb{R}^{N}$ with critical Sobolev exponent, J. Differential Equations 140 (1997), $106-132$.

9. M. Flucher, Variational problems with concentration (Birkhäuser, 1999).

10. M. Guedda and L. Veron, Quasilinear elliptic equations involving critical Sobolev exponents, Nonlinear Analysis 13 (1989), 879-902.

11. Y. Huang, Multiple positive solutions of nonhomogeneous equations involving the p-Laplacian, Nonlinear Analysis 43 (2001), 905-922.

12. M. A. Krasnosel'skii, Topological methods in the theory of nonlinear integral equations (Pergamon Press, Oxford, 1964).

13. P. L. Lions, The concentration-compactness principle in the Calculus of Variations. The limit case, I, II, Rev. Mat. Iberoamericana 1 (1985), 145-201 and 2 (1985), 45-121.

14. A. Marino, La biforcazione nel caso variazionale, Conver. Sem. Mat. Univ. Bari 132 (1973), 14pp.

15. J. Moser, A new proof of De Giorgi's theorem concerning the regularity problem for elliptic differential equations, Commun Pure Appl. Math. 13 (1960), 457-478.

16. P. H. Rabinowitz, Some global results for nonlinear eigenvalue problems, J. Funct. Anal. 7 (1971), 487-513.

17. I. V. Skrypnik, Methods for analysis of nonlinear elliptic boundary value problems, Translations of Mathematical Monographs 139 (Amer. Math. Soc., 1994). 